\title{
miRNAs in Bone Development
}

\author{
Garyfallia Papaioannou*
}

Massachusetts General Hospital and Harvard Medical School, Thier 1101, 50 Blossom Street, Boston MA, 02114, USA

\begin{abstract}
Skeletal development is a multistage process during which mesenchymal progenitor cells undergo proliferation and differentiation and subsequently give rise to bone and cartilage forming cells. Each step is regulated by various transcription factors and signaling molecules. microRNAs are small non-coding RNAs that post-transcriptionally regulate gene expression. Several in vivo and in vitro studies have shown that miRNAs play significant roles in skeletal development. Identifying their functions may give insights into the treatment of developmental disorders of the skeleton. This review summarizes miRNAs that have been shown to participate in various stages of skeletal development by targeting crucial factors.
\end{abstract}

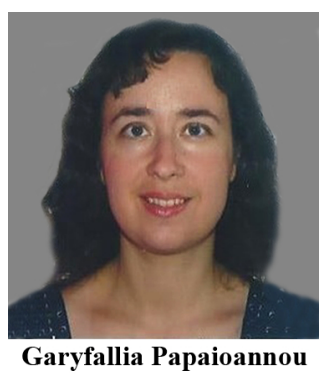

Keywords: Bone, Cartilage, Chondrocytes, Mesenchymal stem cells, mi-RNAs, Osteoblasts, Skeletal development.

\section{INTRODUCTION}

The vertebrate skeleton is composed of cartilage and bone and is derived from three mesodermal lineages. During embryogenesis, cranial neural crest cells form the craniofacial skeleton, paraxial mesodermal cells give rise to the axial skeleton, and lateral plate mesodermal cells form the appendicular skeleton. Migration of these cells to the location of future bone development leads to the formation of mesenchymal condensations. There are several genes that are essential for early patterning of the axial skeleton. Among them, the Hox cluster genes that are expressed in different combinations at particular skeletal axis levels, determine the position of the future vertebrae [1-3].

In locations where endochondral ossification will take place, cells within the mesenchymal condensations first differentiate into chondrocytes, producing a cartilage template (anlage). During endochondral ossification, cartilage is replaced by mineralized bone and bone marrow. Several bones such as the craniofacial bones and the clavicle are formed through direct differentiation of cells in mesenchymal condensations into osteoblasts; this process is called intramembranous ossification. However, the majority of bones are formed by endochondral ossification [4].

Longitudinal bone growth is driven by the growth plate cartilage, where chondrocyte proliferation and differentiation are tightly coordinated. The growth plate consists of three groups of chondrocytes. Slowly proliferating, resting /periarticular chondrocytes differentiate into proliferating chondrocytes that further differentiate into postmitotic hypertrophic chondrocytes. The latter are replaced by mineralized bone [5]. Signaling molecules such as indian hedgehog

*Address correspondence to this author at the Massachusetts General Hospital and Harvard Medical School, Thier 1101, 50 Blossom Street, Boston MA, 02114, USA; Tel: 6177263967;

E-mail: gpapaioannou@mgh.harvard.edu
(Ihh), parathyroid hormone-related peptide (PTHrP), bone morphogenetic proteins (BMPs) and fibroblast growth factors (FGFs) play important roles in the regulation of chondrocyte differentiation. Ihh, expressed by prehypertrophic and hypertrophic chondrocytes, regulates chondrocyte proliferation and differentiation, and expression of PTHrP in resting/periarticular chondrocytes. PTHrP negatively regulates differentiation of prehypertrophic and hypertrophic chondrocytes creating an autoregulatory system. In addition, Ihh couples osteogenesis and chondrogenesis by inducing osteoblast differentiation of perichondrial cells [6]. BMP signaling stimulates differentiation of prechondrogenic cells into chondrocytes and differentiation of proliferating chondrocytes into hypertrophic chondrocytes [7]. FGFs are a large family of secreted proteins with a vast array of biological functions during bone development, including regulation of chondrocyte differentiation and bone formation. They bind to cell surface tyrosine kinase receptors (fibroblast growth factor receptors, FGFRs 1-4), activating multiple signaling pathways such as mitogen-activated protein kinase (MAPK), phosphoinositide 3-kinase (PI3K) and Janus kinase/signal transducer and activator of transcription (JAK/STAT1) [8]. Additionally, transcription factors such as sex determining region Y (SRY) box 9 (Sox9), myocyte enhancer factor 2 (MEF2) family, histone deacetylase 4 (Hdac4) and runt related transcription factor 2 (Runx2) are crucial for chondrocyte differentiation [5]. Sox9 is expressed in osteo-chondro-progenitor cells and upon cell differentiation it is expressed in chondrocytes. It regulates the expression of collagens type II, XI and aggrecan (Acan), being important for chondrocyte function [9]. Runx2 is expressed in mesenchymal condensations, perichondrial cells, hypertrophic chondrocytes and osteoblasts, and one of its functions is to regulate chondrocyte hypertrophy [10-12]. After chondrocytes undergo terminal differentiation (hypertrophy), they mineralize and are replaced by bone. Osteoblasts, the cells that produce mineralized bone matrix, are differentiated from 
local mesenchymal progenitor cells. Ihh, wingless-type MMTV integration site family (Wnt) and Notch signaling pathways as well as Runx2 and Osx (Sp7) transcription factors regulate osteoblast differentiation [13]. Wnt signaling plays an essential role in the regulation of bone mass. In the canonical Wnt signaling pathway, Wnt stabilizes $\beta$-catenin, promoting its nuclear localization. Subsequently, $\beta$-catenin directly regulates gene expression by forming a transcriptional complex with lymphoid enhancer-binding factor 1 (LEF-1) [14]. Notch signaling, through its ligands (Jagged 1,2,Delta-like 1,3,4) and receptors (Notch1-4), suppresses the differentiation of mesechymal precursors into preosteoblasts $[15,16]$. Osx (Sp7) is a zinc finger transcription factor, required for osteoblast differentiation. Osx deficient mice show an absence of osteoblasts and bone formation [17]. The above transcription factors and signaling molecules are essential for the regulation of skeletogenesis. Changes in their expression can significantly influence skeletal development.

Among factors that can alter gene expression are microRNAs (miRNAs). They are single stranded non-coding RNAs, $\sim 22$ nucleotides long that regulate gene expression at the post-transcriptional level. miRNAs are generated after multistep processing of their precursors. Initially, long primary transcripts (pri-miRNAs) are processed into small hairpin RNAs (pre-miRNAs) by the microprocessor complex comprised of Drosha and DGCR8. Pre-miRNAs are further processed by Dicer into their mature form. miRNAs bind mainly to the 3' UTR (untranslated region) of target mRNAs through partial base complementarity. This binding most often results in suppression of translation and mRNA degradation [18]. miRNAs have important roles in diverse cellular functions, such as metabolism, differentiation and apoptosis. Their role in skeletal development has been demonstrated by several studies.

This review will summarize studies on miRNAs that play a role in various stages of skeletal development in vivo and those that have been shown to be related to critical factors for skeletal development in vitro.

\section{MiRNAS IN EARLY SKELETAL DEVELOPMENT}

The overall importance of miRNAs during early skeletal development was first demonstrated by genetic ablation of Dicer.

In mice, the Dicer gene was conditionally deleted in the limb mesenchyme [19] and in chondrocytes [20] causing a global reduction of miRNAs in these tissues. The Cre/loxP recombination system is an effective tool for generating tissue specific-targeted mutants. The enzyme Cre recombinase catalyzes the rearrangement of DNA fragments flanked by two 34bp loxP sites (floxed) [21]. In the limb mesenchyme, floxed Dicer was deleted using a transgene expressing Cre recombinase under the control of a Prx 1 driven enhancer, expressed in the early limb bud mesenchyme and whose activity starts around embryonic day E9.5. Dicer deletion in developing limbs caused a significant reduction of the limb size with increased cell death of limb bud cells. It resulted in morphological malformations such as twisted long bones of arms and legs, and delayed development from cartilage to bone. However, postnatally, all of the differentiated cell types expected in the mature limb bud were present in the Dicer deficient limbs. This suggests that Dicer is required for morphogenesis but not for patterning of the developing limbs [19]. Deletion of Dicer in chondrocytes resulted in significant growth defects and early death by the time of weaning [20]. These mutant mice showed a proportional reduction in skeletal size. The growth defect of the skull and maxilla caused a relative overgrowth of the mandible and lower incisors. A dramatic decrease in proliferation of growth plate chondrocytes, and an increased differentiation of proliferating chondrocytes towards postmitotic hypertrophic chondrocytes were observed [20]. Similar to the Dicer knockout (KO) mice, deletion of Drosha or DGCR8 in chondrocytes caused overall reduction of miRNAs, leading to reduction in proliferating chondrocytes of the growth plate, reduced longitudinal growth of the skull and impaired skeletal development. However, these mice died perinatally [22]. These studies show that miRNAs are necessary for the growth of skeletal tissues during embryogenesis.

miR-196, one of the few miRNAs that are expressed within the Hox clusters, has been shown to target multiple Hox genes, which makes it potentially important for early development [23]. When miR-196 was knocked-down by local antagomir injection in the presomitic mesoderm of chicken stage 11 embryos, HoxB8 expression was expanded anteriorly and the last cervical vertebra was transformed toward a thoracic identity [24]. In many embryos, this was accompanied by the presence of an ectopic rib. Also, when miR-196 was systemically knocked-down, vertebral malformations such as split vertebrae and vertebral fusions were observed. Somites are blocks of mesoderm in the developing vertebrate embryo that give rise to important structures such as skeletal muscle, cartilage, tendons, vertebrae. When miR196 was overexpressed in zebrafish embryos, it reduced total somite number and caused axial abnormalities [25]. miR-196 is an example of a specific miRNA whose deletion leads to abnormalities in early skeleton.

\section{MiRNAS IN POSTNATAL SKELETAL DEVELOP- MENT}

Important in vivo studies have revealed a significant role for miRNAs in growth plate maturation (miR-140 and let-7) and in osteoblast function (miR-2861, miR-3960, miR-182, miR-199, miR-214, miR-17-92 and miR-34) (Table 1).

\section{Role in Growth Plate Maturation}

The chondrocyte specific miRNA, miR-140, is highly expressed in the growth plate. miR-140 deletion in mice causes a mild reduction in the length of endochondral bones and in longitudinal growth of the skull $[26,27]$. Loss of miR-140 led to premature chondrocyte differentiation towards hypertrophic chondrocytes, while the differentiation of resting chondrocytes to proliferating chondrocytes was delayed. The transcription factor Sox 9 was found to regulate miR-140 expression in studies using the chondrocyte cell line, ATDC5 [28], in zebrafish [29] and in mice [30]. miR140 interacts with the $\mathrm{PTHrP} / \mathrm{Hdac} 4$ pathway to control chondrocyte differentiation. Deletion of one allele of $\mathrm{PTHrP}$ or Hdac4 caused growth impairment in mice null for miR-140. MEF2 transcription factors directly stimulate hypertrophic 
Table 1. miRNAs regulating skeletal development, studied in vivo.

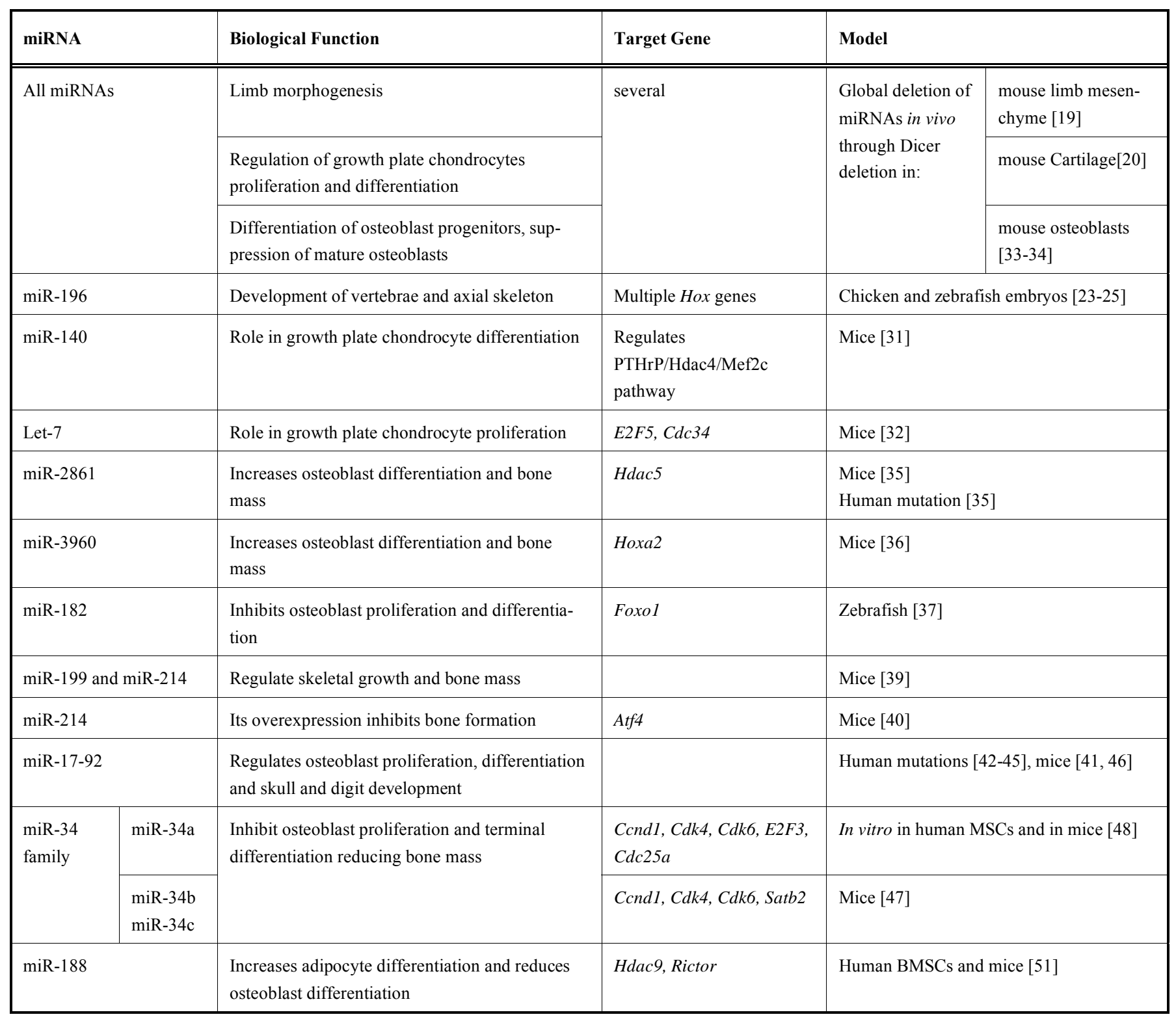

chondrocyte differentiation. MEF2 function is inhibited by Hdac4, the activity of which, is enhanced by PTHrP. Upon miR-140 deletion, there was an increase in Mef2c expression, with normal Hdac4 levels, showing that miR-140 interacts with the PTHrP/Hdac4 pathway at the level of Mef2c [31].

Growth plate chondrocyte proliferation was found to be regulated by let-7 miRNAs. In mice, overexpression of Lin28a, a let-7 inhibitor, resulted in mild growth impairment due to a reduction in chondrocyte proliferation [32]. The cell cycle regulators cell division cycle $34(C d c 34)$ and E2F transcription factor 5 (E2F5) are let-7 targets that mediate this effect. Whereas deficiency of let-7 or miR-140 miRNAs alone caused a mild skeletal phenotype, their combination had a synergistic effect leading to dramatic growth impairment [32]. Thus, let-7 and miR-140 regulate chondrocyte proliferation and differentiation respectively, coordinating skeletal development.

\section{Role in Osteoblasts}

Dicer was deleted in osteoblast progenitors using OsxCre transgenic mice, in which, Cre recombinase is driven by Osx promoter. Dicer loss in these cells caused a reduction in mature osteoblasts and altered texture of mineralized bone matrix without a reduction in bone volume at 4-6 weeks of age [33]. On the contrary, Dicer deletion in mature osteoblasts and osteocytes using osteocalcin-Cre transgenic mice led to increases in bone mass and in expression of the osteoblast markers type I collagen (Collal), Osx ( $S p 7)$, and osteocalcin (bglap) [34]. These studies suggest that miRNAs, overall, promote the differentiation of osteoblast progenitors but suppress the functions of mature osteoblasts.

Regarding specific miRNAs, miR-2861 is expressed mainly in osteoblasts and targets histone deacetylase 5 (Hdac5), an enzyme that regulates various cellular proteins, including Runx2 and Mef2 transcription factors [35]. miR- 
2861, by reducing Hdac5 expression, increased Runx2 expression to enhance osteoblast differentiation. Bone mineral density was reduced in mice treated with antisense miR2861, while a mutation in the miR-2861 gene was found in some patients with adolescent osteoporosis [35]. These findings suggest that normal function of miR-2861 is important for postnatal skeletal development and maintenance of bone mass. miR-2861 is located in the promoter region of cyclin dependent kinase 9 gene ( $C d k 9)$, a gene that controls cell cycle. Thus, another explanation for the osteoporosis phenotype is that the above mutation affects $\mathrm{Cdk} 9$ function, altering normal osteoblast proliferation. A similar role was attributed to miR-3960, encoded by the same transcript. miR3960 was shown to target Hoxa2, to inhibit Runx2 and thereby promoted osteoblast differentiation [36]. A feedback loop, in which Runx2 increases the expression of these two miRNAs by binding to their common promoter, was demonstrated in the same study.

miR-182, inhibits osteoblast proliferation and differentiation. It was shown in zebrafish to inhibit bone formation [37]. The transcription factor Foxo1, which positively regulates bone mass, was suppressed by miR-182.

miR-199 and miR-214 are encoded in the dynamin 3 (Dnm3) gene locus, and transcribed in the opposite direction to Dnm3. These miRNAs are mostly co-regulated as a unit, with some exceptions such as their differential expression in the notochord. On the other hand, these miRNAs are differentially regulated from their host $d n m$ gene [38]. In mice, deletion of miR-199 and miR-214 caused growth retardation, craniofacial and vertebral hypoplasia as well as osteopenia [39]. These miRNAs are expressed in mesenchymal cells, perichondrial cells and periarticular chondrocytes. Overexpression of miR-214 was shown to reduce bone formation in mice. Atf4, encoding for a transcription factor required for osteoblast function, was identified as a target of miR-214 [40].

Skeletal developmental defects in humans can be caused by mutations in the miRNA cluster miR-17-92. It is abundantly expressed in osteoprogenitor and embryonic stem cells, but decreases upon osteoblast differentiation [41]. Feingold syndrome type II, a brachy-syndactyly syndrome, is caused by deletion of the miR-17-92 gene [42, 43]. Duplication of this gene has also been found in patients showing skeletal overgrowth, macrocephaly, and polydactyly in one family [44]. miR-17-92 microduplication was also found in another family in which affected patients showed short stature and brachydactyly [45]. Heterozygous deletion of the miR-17-92 cluster in mice caused reduction of skeletal growth and delayed ossification of digital and skull bones [42]. The reduction of miR-17-92 levels caused impaired osteoblast proliferation and differentiation and, reduced bone mass in mice [41]. Mice lacking miR-92a show growth retardation beginning around embryonic day E15.5 that leads to postnatal reduction in body weight compared to control mice. These mice have reduced size of long bones and skull, consistent with an overall reduction of body size. However, the bone density of miR-92a-null mice is normal. A mild reduction in the relative length of the $5^{\text {th }}$ mesophalanx of the forelimbs is observed. Thus, miR-92, among miR-17-92 miRNAs may play a particularly important role in development of these bones [46].
miRNA profiling in differentiated mouse calvarial osteoblasts, showed that miR-34b and miR-34c were significantly upregulated ( $\sim 1.8$ fold) and had relatively restricted expression in osteoblasts [47]. Trangenic mice overexpressing miR-34c had a low bone mass phenotype at 3 months of age. The number of osteoblasts and bone formation rate were reduced. Mice in which both miR-34b and miR-34c were deleted in mature osteoblasts using CollCre transgenic mice showed increases in bone mass, osteoblast proliferation and bone formation rate. Cell cycle regulators, including cyclin D1 (Ccnd1) and cyclin dependent kinases Cdk4 and Cdk6 were identified as targets of miR $-34 b$ and c. Additionally, $\mathrm{miR}-34 \mathrm{~b} / \mathrm{c}$ deletion in osteoblasts increased osteoblast differentiation in vivo and ex vivo. Satb2 (special AT-rich sequence binding protein 2), a protein that promotes osteoblast differentiation, was found to be the target mediating this effect. Thus, miR34b/c regulate skeletogenesis by inhibiting osteoblast proliferation and terminal differentiation. [47]. On the other hand, an ex vivo study, demonstrated that miR-34a was significantly upregulated upon differentiation of human mesenchymal stem cells (MSCs) into osteoblasts. miR-34b showed a smaller increase but miR-34c was not detectable [48]. miR-34a overexpression in human MSCs inhibited osteoblast differentiation and mineralization. miR-34a targeted Jag1, encoding for a Notch ligand. Also, miR-34a targeted the cell cycle regulators, Ccnd1, Cdk4 and $C d k 6$, E2F transcription factor $3(E 2 F 3)$ and cell division cycle $25 \mathrm{~A}$ $(\mathrm{Cdc} 25 \mathrm{a})$, to decrease osteoblast proliferation. miR-34a overexpression in MSCs subcutaneously implanted into immune deficient mice inhibited heterotopic bone formation in mice whereas anti-miR-34a overexpression had the opposite effect [48]. These studies suggest that miR-34 family miRNAs are negative regulators of bone development.

Osteogenesis is reduced upon aging, while fat accumulation in the bone marrow increases $[49,50]$. A recent in vivo study showed that aging induces the expression of miR-188 in bone marrow MSCs (BMSCs) of mice and humans [51]. miR$188 \mathrm{KO}$ mice showed less bone marrow adiposity and a reduction in age-related bone loss. The opposite phenotype was observed when miR-188 was overexpressed in osteoprogenitors or in BMSCs in mice. Hdac9 and RPTOR-independent companion of MTOR complex 2 (Rictor) were identified as direct targets of miR-188. Importantly, injection of antagomiR-188 into bone marrow stimulated bone formation and decreased marrow fat in aged mice [51]. These findings suggest that miR-188 is an important regulator of the age-related switch between osteoblast and adipocyte differentiation.

\section{OTHER MIRNAS (STUDIED IN VITRO)}

Several other miRNAs were shown to regulate the behavior of chondrocytes, osteoblasts and their progenitors in $v i$ tro. In several studies, miRNA profiling and comparison of miRNA expression patterns were performed between cells of different stages of differentiation. These screening in vitro experiments identified microRNAs potentially important for skeletal development.

\section{miRNAs Regulating Chondrocytes and Their Progenitors In Vitro}

miR-675, highly expressed in human and murine articular cartilage, upregulated Col2al expression in human articu- 
lar chondrocytes [52]. miR-1247, strongly expressed in mouse cartilage was found to target Sox9 [53]. Overexpression of miR-145, another miRNA shown to target Sox9, decreased the expression of chondrocyte marker genes during chondrocytic differentiation of a murine embryonic mesenchymal cell line (C3H10T1/2 cells). The downregulated chondrocyte markers included Col2a1, Acan, Col9a1, Coll1a1 and cartilage oligomeric matrix protein (Comp). miR-145 was downregulated during chondrocyte differentiation [54]. Similarly, miR-145 was found to regulate human articular chondrocyte function [55]. In rat articular cartilage, miR-337 was highly expressed at birth, but at postnatal days 21 and 42 it was significantly decreased. miR-337 overexpression in a human chondrocyte cell line inhibited chondrocyte differentiation. Tgfbr2, encoding a receptor of transforming growth factor beta, was identified as a miR-337 target [56]. In chicken limb mesenchymal cells miR-375 decreased upon chondrocyte differentiation, and inhibition of miR-375 promoted chondrogenesis. Cadherin-7, a calcium dependent cell to cell adhesion glycoprotein, was found to be a direct miR-375 target [57]. MSC chondrocyte differentiation is inhibited by miR-449. In human bone marrow MSCs and in human chondrosarcoma cell lines miR-449 reduced the expression of Col2a1 and Sox9 and proteoglycan synthesis. These actions were mediated by suppression of Lefl, encoding a transcription factor that mediates Wnt signaling [58]. During human MSC differentiation into chondrocytes, miR-574-3p was significantly upregulated. It was shown that Sox 9 increases the transcription of miR-574-3p by binding to its promoter, while retinoid $\mathrm{X}$ receptor alpha (Rxra) was found to be a direct target of this miRNA [59]. miR335-5p was also upregulated during chondrocyte differentiation of MSCs, promoting chondrogenesis. The negative regulators of Sox 9, Rock1 and Daaml were targeted by miR335 [60]. A recent study identified miR-29a, which had previously been reported to regulate osteoblast function, as an inhibitor of chondrogenesis in human MSCs. Direct targeting of Foxo3 (forkhead box O3) mediated this effect [61].

\section{microRNAs Regulating Osteoblasts and Their Progeni- tors In Vitro}

In human embryonic femurs (7-9 weeks post conception), miRNAs are differentially expressed in epiphyseal and diaphyseal cell populations [62]. Upon further analysis of miRNA expression, miR-146b, miR-301 and miR-138 showed greater expression levels in epiphyseal cells than in diaphyseal cells, while miR-143, miR-145, miR-146a and miR-34a showed greater expression in diaphyseal cells. Among these miRNAs, function of miR-146a was further investigated in vitro. miR-146a was found to target Smad3, which increases Runx2 expression to promote osteogenesis [62]. miR-146a was previously reported to be abundantly expressed in umbilical cord MSCs [63]. miR-146a knockdown in these cells, inhibited their proliferation and promoted their migration [63]. Cxcl12 (chemokine ligand 12) and Sike1, an I-kappa-B kinase epsilon (IKKe) suppressor were determined as targets of miR-146 in the umbilical cord MSCs [63]. Thus, there may be a broader role for miR-146 in development. Another miRNA found to increase Runx2 levels is miR-15b. In human bone marrow MSCs, it targeted Smurfl encoding a protein that facilitates Runx2 degradation
[64]. In contrast, miR-338-3p, which was downregulated during osteoblast differentiation of BMSCs, inhibited osteoblast differentiation by targeting Runx2 and Fgfr2 [65].

Several miRNAs regulate Wnt signaling. Among them are the miR-29 family miRNAs, which are highly conserved among vertebrates [66]. miR-29a suppressed the expression of the Wnt signaling inhibitors Dickkopf-related protein 1 $(D k k 1)$, Kremen2 and secreted frizzled related protein 2 (Sfrp2), inducing osteoblast differentiation in primary human osteoblast cultures. Wnt signaling induced miR-29a expression, thus likely creating a positive feedback loop [67]. Rats treated with a miR-29a inhibitor showed a significant reduction in bone mass [68]. In a recent study, miR-29a was also studied in a fish bone-derived cell line (ABS 15 cells), where it was shown to increase $\beta$-catenin levels and mineralization. The transcript of the Sparc gene, encoding osteonectin, a non-collagen ECM protein, was determined as a target of miR-29a [66]. miR-29b was upregulated during osteoblast differentiation of primary rat calvaria osteoblasts and was found to target Hdac4, Tgfb3 (TGF-beta3), Acvr2 $\alpha$ (activin receptor II $\alpha$ ), Ctnnblipl (catenin beta interacting protein 1) and Dusp2 (dual specificity phosphatase 2), factors that inhibit osteoblast differentiation [69]. miR-29b expression was found to be reduced in patients with osteogenesis imperfecta [70]. miR-335-5p, discussed earlier for its role in chondrogenesis, was found in osteoblasts of mouse embryos. It suppressed Dkkl and enhanced osteogenesis. [71]. In a different study, miRNA expression was analyzed after Wnt3a stimulation in vitro, to investigate the effects of Wnt signaling on miRNA expression in human MSCs. miR141-3p was downregulated upon Wnt signaling activation, while miR-141-3p overexpression inhibited Wnt3a activity and cell proliferation. It targeted Cdc25a, thus regulating cell proliferation [72]. Also, in the same study, reduction of Cdc25a was considered to mediate an inhibitory effect on osteoblast differentiation of human MSCs. In search of regulators of the Wnt signaling pathway, Wang et al. performed microarray analysis to screen for miRNAs whose expression changed during osteoblast differentiation of human BMSCs [73]. miR-346 was identified as a promoter of osteoblast differentiation and upon its overexpression, there was a significant increase in osteoblast markers. It was shown to directly inhibit $G s k 3 b[73]$.

BMP signaling is another important pathway regulated by miRNAs in bone. miR-497 195 cluster miRNAs are members of the miR-15 family. It is broadly expressed in tissues like lung, skin, muscle, heart and bone. Its levels were examined in mouse tibia and calvaria bones at different ages (Postnatal days P0-P42). miR-497-195 cluster miRNAs were strongly upregulated with age during postnatal bone development [74]. They were also upregulated upon final differentiation of primary calvarial osteoblasts in vitro. miR195 acted as an intracellular antagonist of BMP signaling and inhibited osteoblast differentiation in vitro. Microarray and bioinformatics analysis revealed that several mRNAs encoding molecules related to BMP signaling are targets of miR-195; these genes include Acvrl (activin A receptor type 1), Bmprla (bone morphogenetic protein receptor type 1A), Tgfbr3 (transforming growth factor, beta receptor III), Smad5 [74]. Another miRNA that regulates BMP signaling is miR-542-3p. miR-542-3p overexpression in mouse cal- 
varial osteoblasts in vitro inhibited differentiation [75]. Bmp7 was identified as a direct target of miR-542-3p. Moreover, transfection with miR-542-3p mimic led to reductions in activity of the PI3K/Akt pathway and expression of survivin (an apoptosis inhibitor), causing caspase 3 activation and osteoblast apoptosis. Inhibition of osteoblast proliferation was also observed. In vivo treatment of ovariectomized mice with anti-miR-542-3p increased bone mass [75]. miR-140, a miRNA discussed earlier for its role in growth plate development, inhibited osteoblast lineage commitment in human MSCs by directly repressing Bmp2 [76].

\section{FUTURE PERSPECTIVES}

miRNAs have therefore, important roles in various stages of skeletal development, miRNAs and their inhibitors may be used as therapeutic options to treat skeletal disorders. miRNA mimics increase the levels of beneficial miRNAs, whereas miRNA inhibitors (antisense oligonucleotides) inhibit disease promoting miRNAs through complementary base pairing. Animal studies have shown promising results but there are still many challenges in applying miRNA based therapy in human diseases [77]. miRNAs have, often, multiple targets something that may cause several side effects. Efficient local tissue delivery and avoidance of toxicity of the nucleotide analogs are also important aspects for consideration [77]. Understanding the role of each miRNA and the effects of individual or simultaneous miRNA manipulation in vivo will facilitate the development of effective and safe miRNA-mediated therapy for developmental disorders of the skeleton.

\section{CONCLUSIONS}

Bone development is controlled by several molecules and signaling pathways and miRNAs play significant roles in this regulation. Although some in vivo studies have demonstrated important roles of miRNAs, the majority of studies has been performed in vitro, using cultured primary cells or cell lines. It is crucial to confirm these results in vivo in order to understand the physiological roles of miRNAs in bone development and identify their significance in humans. Further work is needed to translate these findings into clinical applications.

\section{CONFLICT OF INTEREST}

The author(s) confirm that this article content has no conflict of interest.

\section{ACKNOWLEDGEMENTS}

The author acknowledges Dr Tatsuya Kobayashi and Dr Eva S Liu for helpful discussion and editing.

\section{REFERENCES}

Kessel, M.; Gruss, P. Homeotic transformations of murine vertebrae and concomitant alteration of Hox codes induced by retinoic acid. Cell, 1991, 67 (1), 89-104.

[2] Krumlauf, R. Hox genes in vertebrate development. Cell, 1994, 78 (2), 191-201.

[3] Chen, F.; Capecchi, M.R. Targeted mutations in hoxa-9 and hoxb-9 reveal synergistic interactions. Dev. Biol., 1997, 181 (2), 186-196.
Rev. Cell Dev. Biol., 2000, 16, 191-220.

[5] Kronenberg, H.M. Developmental regulation of the growth plate. Nature, 2003, 423(6937), 332-336.

[6] Long, F.; Chung, U.I.; Ohba, S.; McMahon, J.; Kronenberg, H.M.; McMahon, A.P. Ihh signaling is directly required for the osteoblast lineage in the endochondral skeleton. Development, 2004, 131(6), 1309-1318.

[7] Kobayashi, T.; Lyons, K.M.; McMahon, A.P.; Kronenberg, H.M. BMP signaling stimulates cellular differentiation at multiple steps during cartilage development. Proc. Natl. Acad. Sci. U S A, 2005, 102(50), 18023-18027.

[8] Itoh, N.; Ornitz, D.M. Fibroblast growth factors: from molecular evolution to roles in development, metabolism and disease. J. Biochem., 2011, 149(2), 121-130.

[9] Lefebvre, V.; Huang, W.; Harley, V.R.; Goodfellow, P.N.; de Crombrugghe, B. SOX9 is a potent activator of the chondrocytespecific enhancer of the pro alpha1(II) collagen gene. Mol. Cell. Biol., 1997, 17(4), 2336-2346.

[10] Yoshida, C.A.; Yamamoto, H.; Fujita, T.; Furuichi, T.; Ito, K.; Inoue, K.; Yamana, K.; Zanma, A.; Takada, K.; Ito, Y.; Komori, T. Runx2 and Runx3 are essential for chondrocyte maturation, and Runx2 regulates limb growth through induction of Indian hedgehog. Genes Dev., 2004, 18(8), 952-963.

[11] Takeda, S.; Bonnamy, J.P.; Owen, M.J.; Ducy, P.; Karsenty, G. Continuous expression of Cbfa1 in nonhypertrophic chondrocytes uncovers its ability to induce hypertrophic chondrocyte differentiation and partially rescues Cbfa1-deficient mice. Genes Dev., 2001, 15(4), 467-481.

[12] Otto, F.; Thornell, A.P.; Crompton, T.; Denzel, A.; Gilmour, K.C.; Rosewell, I.R.; Stamp, G.W.; Beddington, R.S.; Mundlos, S.; Olsen, B.R.; Selby, P.B.; Owen, M.J. Cbfa1, a candidate gene for cleidocranial dysplasia syndrome, is essential for osteoblast differentiation and bone development. Cell, 1997, 89(5), 765-771.

[13] Karsenty, G.; Kronenberg, H. M.; Settembre, C. Genetic control of bone formation. Annu Rev Cell Dev. Biol., 2009, 25, 629-648.

[14] Canalis, E. Wnt signalling in osteoporosis: mechanisms and novel therapeutic approaches. Nat Rev. Endocrinol., 2013, 9(10), 575583.

[15] Rebay, I.; Fleming, R.J.; Fehon, R.G.; Cherbas, L.; Cherbas, P.; Artavanis-Tsakonas, S. Specific EGF repeats of Notch mediate interactions with Delta and Serrate: implications for Notch as a multifunctional receptor. Cell, 1991, 67(4), 687-699.

[16] Hilton, M.J.; Tu, X.; Wu, X.; Bai, S.; Zhao, H.; Kobayashi, T.; Kronenberg, H. M.; Teitelbaum, S. L.; Ross, F. P.; Kopan, R.; Long, F. Notch signaling maintains bone marrow mesenchymal progenitors by suppressing osteoblast differentiation. Nat. Med., 2008, 14 (3), 306-314.

[17] Nakashima, K.; Zhou, X.; Kunkel, G.; Zhang, Z.; Deng, J.M.; Behringer, R.R.; de Crombrugghe, B. The novel zinc fingercontaining transcription factor osterix is required for osteoblast differentiation and bone formation. Cell, 2002, 108(1), 17-29.

[18] Tetreault, N.; De Guire, V. miRNAs: their discovery, biogenesis and mechanism of action. Clin. Biochem., 2013, 46(10-11), 842845 .

[19] Harfe, B.D.; McManus, M.T.; Mansfield, J.H.; Hornstein, E.; Tabin, C.J. The RNaseIII enzyme Dicer is required for morphogenesis but not patterning of the vertebrate limb. Proc. Natl. Acad. Sci. U S A, 2005, 102(31), 10898-10903.

[20] Kobayashi, T.; Lu, J.; Cobb, B.S.; Rodda, S.J.; McMahon, A.P.; Schipani, E.; Merkenschlager, M.; Kronenberg, H.M. Dicerdependent pathways regulate chondrocyte proliferation and differentiation. Proc. Natl. Acad. Sci. U S A, 2008, 105(6), 19491954.

[21] Sauer, B.; Henderson, N. Site-specific DNA recombination in mammalian cells by the Cre recombinase of bacteriophage P1. Proc. Natl. Acad. Sci. U S A, 1988, 85(14), 5166-5170.

[22] Kobayashi, T.; Papaioannou, G.; Mirzamohammadi, F.; Kozhemyakina, E.; Zhang, M.; Blelloch, R.; Chong, M.W. Early postnatal ablation of the microRNA-processing enzyme, Drosha, causes chondrocyte death and impairs the structural integrity of the articular cartilage. Osteoarthritis Cartilage, 2015, 23(7),1214-20.

[23] Yekta, S.; Shih, I.H.; Bartel, D.P. MicroRNA-directed cleavage of HOXB8 mRNA. Science, 2004, 304(5670), 594-596.

[24] McGlinn, E.; Yekta, S.; Mansfield, J.H.; Soutschek, J.; Bartel, D. P.; Tabin, C.J. In ovo application of antagomiRs indicates a role for miR-196 in patterning the chick axial skeleton through Hox gene 
regulation. Proc. Natl. Acad. Sci. US A, 2009, 106(44), 1861018615.

[25] He, X.; Yan, Y.L.; Eberhart, J.K.; Herpin, A.; Wagner, T.U.; Schartl, M.; Postlethwait, J. H. miR-196 regulates axial patterning and pectoral appendage initiation. Dev. Biol., 2011, 357(2), 463477.

[26] Miyaki, S.; Sato, T.; Inoue, A.; Otsuki, S.; Ito, Y.; Yokoyama, S.; Kato, Y.; Takemoto, F.; Nakasa, T.; Yamashita, S.; Takada, S.; Lotz, M. K.; Ueno-Kudo, H.; Asahara, H. MicroRNA-140 plays dual roles in both cartilage development and homeostasis. Genes Dev., 2010, 24 (11), 1173-1185.

[27] Nakamura, Y.; Inloes, J. B.; Katagiri, T.; Kobayashi, T. Chondrocyte-specific microRNA-140 regulates endochondral bone development and targets Dnpep to modulate bone morphogenetic protein signaling. Mol. Cell Biol., 2011, 31 (14), 3019-3028.

[28] Yang, J.; Qin, S.; Yi, C.; Ma, G.; Zhu, H.; Zhou, W.; Xiong, Y.; Zhu, X.; Wang, Y.; He, L.; Guo, X. MiR-140 is co-expressed with Wwp2-C transcript and activated by Sox9 to target Sp1 in maintaining the chondrocyte proliferation. FEBS Lett, 2011, 585(19), 2992-2997.

[29] Nakamura, Y.; He, X.; Kato, H.; Wakitani, S.; Kobayashi, T.; Watanabe, S.; Iida, A.; Tahara, H.; Warman, M.L.; Watanapokasin, R.; Postlethwait, J.H. Sox9 is upstream of microRNA-140 in cartilage. Appl. Biochem. Biotechnol., 2012, 166 (1), 64-71.

[30] Yamashita, S.; Miyaki, S.; Kato, Y.; Yokoyama, S.; Sato, T.; Barrionuevo, F.; Akiyama, H.; Scherer, G.; Takada, S.; Asahara, H. LSox5 and Sox6 proteins enhance chondrogenic miR-140 microRNA expression by strengthening dimeric Sox9 activity. J. Biol. Chem., 2012, 287(26), 22206-22215.

[31] Papaioannou, G.; Mirzamohammadi, F.; Lisse, T. S.; Nishimori, S.; Wein, M. N.; Kobayashi, T. MicroRNA-140 provides robustness to the regulation of hypertrophic chondrocyte differentiation by the PTHrP-HDAC4 pathway. J. Bone Miner. Res., 2015, 30(6), 104452.

[32] Papaioannou, G.; Inloes, J.B.; Nakamura, Y.; Paltrinieri, E.; Kobayashi, T. let-7 and miR-140 microRNAs coordinately regulate skeletal development. Proc. Natl. Acad. Sci. U S A, 2013, 110(35), E3291-3300.

[33] Raaijmakers, M.H.; Mukherjee, S.; Guo, S.; Zhang, S.; Kobayashi, T.; Schoonmaker, J.A.; Ebert, B.L.; Al-Shahrour, F.; Hasserjian, R. P.; Scadden, E.O.; Aung, Z.; Matza, M.; Merkenschlager, M.; Lin, C.; Rommens, J.M.; Scadden, D.T. Bone progenitor dysfunction induces myelodysplasia and secondary leukaemia. Nature, 2010, 464(7290), 852-857.

[34] Gaur, T.; Hussain, S.; Mudhasani, R.; Parulkar, I.; Colby, J.L.; Frederick, D.; Kream, B.E.; van Wijnen, A.J.; Stein, J.L.; Stein, G. S.; Jones, S.N.; Lian, J.B. Dicer inactivation in osteoprogenitor cells compromises fetal survival and bone formation, while excision in differentiated osteoblasts increases bone mass in the adult mouse. Dev. Biol., 2010, 340(1), 10-21.

[35] Li, H.; Xie, H.; Liu, W.; Hu, R.; Huang, B.; Tan, Y.F.; Xu, K.; Sheng, Z.F.; Zhou, H.D.; Wu, X.P.; Luo, X.H. A novel microRNA targeting HDAC5 regulates osteoblast differentiation in mice and contributes to primary osteoporosis in humans. J. Clin. Invest., 2009, 119 (12), 3666-3677.

[36] Hu, R.; Liu, W.; Li, H.; Yang, L.; Chen, C.; Xia, Z.Y.; Guo, L.J.; Xie, H.; Zhou, H.D.; Wu, X.P.; Luo, X.H. A Runx2/miR3960/miR-2861 regulatory feedback loop during mouse osteoblast differentiation. J. Biol. Chem., 2011, 286(14), 12328-12339.

[37] Kim, K.M.; Park, S.J.; Jung, S.H.; Kim, E.J.; Jogeswar, G.; Ajita, J.; Rhee, Y.; Kim, C.H.; Lim, S.K. miR-182 is a negative regulator of osteoblast proliferation, differentiation, and skeletogenesis through targeting FoxO1. J. Bone Miner. Res., 2012, 27(8), 16691679.

[38] Desvignes, T.; Contreras, A.; Postlethwait, J.H. Evolution of the miR199-214 cluster and vertebrate skeletal development. RNA Biol., 2014, 11(4), 281-294.

[39] Watanabe, T.; Sato, T.; Amano, T.; Kawamura, Y.; Kawamura, N.; Kawaguchi, H.; Yamashita, N.; Kurihara, H.; Nakaoka, T. Dnm3os, a non-coding RNA, is required for normal growth and skeletal development in mice. Dev. Dyn., 2008, 237(12), 37383748 .

[40] Wang, X.; Guo, B.; Li, Q.; Peng, J.; Yang, Z.; Wang, A.; Li, D.; Hou, Z.; Lv, K.; Kan, G.; Cao, H.; Wu, H.; Song, J.; Pan, X.; Sun, Q.; Ling, S.; Li, Y.; Zhu, M.; Zhang, P.; Peng, S.; Xie, X.; Tang, T.; Hong, A.; Bian, Z.; Bai, Y.; Lu, A.; Li, Y.; He, F.; Zhang, G.;
Li, Y. miR-214 targets ATF4 to inhibit bone formation. Nat. Med., 2013, 19(1), 93-100.

[41] Zhou, M.; Ma, J.; Chen, S.; Chen, X.; Yu, X. MicroRNA-17-92 cluster regulates osteoblast proliferation and differentiation. Endocrine, 2014, 45(2), 302-10.

[42] de Pontual, L.; Yao, E.; Callier, P.; Faivre, L.; Drouin, V.; Cariou, S.; Van Haeringen, A.; Genevieve, D.; Goldenberg, A.; Oufadem, M.; Manouvrier, S.; Munnich, A.; Vidigal, J. A.; Vekemans, M.; Lyonnet, S.; Henrion-Caude, A.; Ventura, A.; Amiel, J. Germline deletion of the miR-17 approximately 92 cluster causes skeletal and growth defects in humans. Nat. Genet., 2011, 43(10), 1026-1030.

[43] Tassano, E.; Di Rocco, M.; Signa, S.; Gimelli, G. De novo 13q31.1-q32.1 interstitial deletion encompassing the miR-17-92 cluster in a patient with Feingold syndrome-2. Am. J. Med. Genet. A, 2013, 161A(4), 894-896.

[44] Kannu, P.; Campos-Xavier, A. B.; Hull, D.; Martinet, D.; Ballhausen, D.; Bonafe, L. Post-axial polydactyly type A2, overgrowth and autistic traits associated with a chromosome $13 \mathrm{q} 31.3$ microduplication encompassing miR-17-92 and GPC5. Eur. J. Med. Genet., 2013, 56(8), 452-457.

[45] Hemmat, M.; Rumple, M.J.; Mahon, L.W.; Strom, C.M.; Anguiano, A.; Talai, M.; Nguyen, B.; Boyar, F. Z. Short stature, digit anomalies and dysmorphic facial features are associated with the duplication of miR-17 92 cluster. Mol. Cytogenet., 2014, 7(1), 27.

[46] Penzkofer, D.; Bonauer, A.; Fischer, A.; Tups, A.; Brandes, R. P.; Zeiher, A.M.; Dimmeler, S. Phenotypic characterization of miR92a-/- mice reveals an important function of miR-92a in skeletal development. PLoS One, 2014, 9(6), e101153.

[47] Wei, J.; Shi, Y.; Zheng, L.; Zhou, B.; Inose, H.; Wang, J.; Guo, X. E.; Grosschedl, R.; Karsenty, G. miR-34s inhibit osteoblast proliferation and differentiation in the mouse by targeting SATB2. $J$. Cell. Biol., 2012, 197(4), 509-521.

[48] Chen, L.; Holmstrom, K.; Qiu, W.; Ditzel, N.; Shi, K.; Hokland, L. B.; Kassem, M. MicroRNA-34a Inhibits osteoblast differentiation and in vivo bone formation of human stromal stem cells. Stem Cells, 2014, 32(4), 902-12.

[49] Marie, P.J.; Kassem, M. Extrinsic mechanisms involved in agerelated defective bone formation. J. Clin. Endocrinol. Metab., 2011, 96(3), 600-609.

[50] Bethel, M.; Chitteti, B.R.; Srour, E.F.; Kacena, M.A. The changing balance between osteoblastogenesis and adipogenesis in aging and its impact on hematopoiesis. Curr. Osteoporos. Rep., 2013, 11(2), 99-106.

[51] Li, C.J.; Cheng, P.; Liang, M.K.; Chen, Y.S.; Lu, Q.; Wang, J.Y.; Xia, Z.Y.; Zhou, H.D.; Cao, X.; Xie, H.; Liao, E.Y.; Luo, X.H. MicroRNA-188 regulates age-related switch between osteoblast and adipocyte differentiation. J. Clin. Invest., 2015, 125(4), 1509-1522.

[52] Dudek, K.A.; Lafont, J.E.; Martinez-Sanchez, A.; Murphy, C.L. Type II collagen expression is regulated by tissue-specific miR-675 in human articular chondrocytes. J. Biol. Chem., 2010, 285(32), 24381-24387.

[53] Martinez-Sanchez, A.; Murphy, C. L. miR-1247 functions by targeting cartilage transcription factor SOX9. J. Biol. Chem., 2013, 288(43), 30802-30814

[54] Yang, B.; Guo, H.; Zhang, Y.; Chen, L.; Ying, D.; Dong, S. MicroRNA-145 regulates chondrogenic differentiation of mesenchymal stem cells by targeting Sox9. PLoS One, 2011, 6(7), e21679.

[55] Martinez-Sanchez, A.; Dudek, K.A.; Murphy, C. L. Regulation of human chondrocyte function through direct inhibition of cartilage master regulator SOX9 by microRNA-145 (miRNA-145). J. Biol. Chem., 2012, 287(2), 916-924.

[56] Zhong, N.; Sun, J.; Min, Z.; Zhao, W.; Zhang, R.; Wang, W.; Tian, J.; Tian, L.; Ma, J.; Li, D.; Han, Y.; Lu, S. MicroRNA-337 is associated with chondrogenesis through regulating TGFBR2 expression. Osteoarthritis Cartilage, 2012, 20(6), 593-602.

[57] Song, J.; Kim, D.; Chun, C.H.; Jin, E.J. MicroRNA-375, a new regulator of cadherin-7, suppresses the migration of chondrogenic progenitors. Cell Signal., 2013, 25(3), 698-706.

[58] Paik, S.; Jung, H.S.; Lee, S.; Yoon, D.S.; Park, M.S.; Lee, J.W. miR-449a regulates the chondrogenesis of human mesenchymal stem cells through direct targeting of lymphoid enhancer-binding factor-1. Stem Cells Dev., 2012, 21(18), 3298-3308.

[59] Guerit, D.; Philipot, D.; Chuchana, P.; Toupet, K.; Brondello, J.M.; Mathieu, M.; Jorgensen, C.; Noel, D. Sox9-regulated miRNA-574$3 \mathrm{p}$ inhibits chondrogenic differentiation of mesenchymal stem 
cells. PLoS One, 2013, 8(4), e62582.

[60] Lin, X.; Wu, L.; Zhang, Z.; Yang, R.; Guan, Q.; Hou, X.; Wu, Q. MiR-335-5p Promotes Chondrogenesis in Mouse Mesenchymal Stem Cells and is Regulated Through Two Positive Feedback Loops. J. Bone Miner. Res., 2013, 29(7), 1575-85.

[61] Guerit, D.; Brondello, J.M.; Chuchana, P.; Philipot, D.; Toupet, K.; Bony, C.; Jorgensen, C.; Noel, D. FOXO3A regulation by miRNA29a Controls chondrogenic differentiation of mesenchymal stem cells and cartilage formation. Stem Cells Dev., 2014, 23(11), 11951205.

[62] Cheung, K.S.; Sposito, N.; Stumpf, P.S.; Wilson, D.I.; SanchezElsner, T.; Oreffo, R.O. MicroRNA-146a regulates human foetal femur derived skeletal stem cell differentiation by down-regulating SMAD2 and SMAD3. PLoS One, 2014, 9(6), e98063.

[63] Hsieh, J.Y.; Huang, T.S.; Cheng, S.M.; Lin, W.S.; Tsai, T.N.; Lee, O.K.; Wang, H.W. miR-146a-5p circuitry uncouples cell proliferation and migration, but not differentiation, in human mesenchymal stem cells. Nucleic Acids Res., 2013, 41(21), 9753-9763.

[64] Vimalraj, S.; Partridge, N.C.; Selvamurugan, N. A positive role of microRNA-15b on regulation of osteoblast differentiation. J. Cell Physiol., 2014, 229(9), 1236-1244.

[65] Liu, H.; Sun, Q.; Wan, C.; Li, L.; Zhang, L.; Chen, Z. MicroRNA338-3p regulates osteogenic differentiation of mouse bone marrow stromal stem cells by targeting Runx2 and Fgfr2. J. Cell Physiol., 2014, 229(10), 1494-1502.

[66] Roberto, V.P.; Tiago, D.M.; Silva, I.A.; Cancela, M.L. MiR-29a is an enhancer of mineral deposition in bone-derived systems. Arch. Biochem. Biophys., 2014, 564, 173-183.

[67] Kapinas, K.; Kessler, C.; Ricks, T.; Gronowicz, G.; Delany, A.M. miR-29 modulates Wnt signaling in human osteoblasts through a positive feedback loop. J. Biol. Chem., 2010, 285(33), 2522125231.

[68] Wang, F. S.; Chuang, P. C.; Lin, C. L.; Chen, M. W.; Ke, H. J.; Chang, Y.H.; Chen, Y.S.; Wu, S.L.; Ko, J.Y. MicroRNA-29a protects against glucocorticoid-induced bone loss and fragility in rats by orchestrating bone acquisition and resorption. Arthritis Rheum., 2013, 65 (6), 1530-1540.
[69] Li, Z.; Hassan, M.Q.; Jafferji, M.; Aqeilan, R.I.; Garzon, R.; Croce, C.M.; van Wijnen, A.J.; Stein, J.L.; Stein, G.S.; Lian, J.B. Biological functions of miR-29b contribute to positive regulation of osteoblast differentiation. J. Biol. Chem., 2009, 284(23), 1567615684.

[70] Kaneto, C.M.; Lima, P.S.; Zanette, D.L.; Prata, K.L.; Pina Neto, J. M.; de Paula, F.J.; Silva, W.A. Jr. COL1A1 and miR-29b show lower expression levels during osteoblast differentiation of bone marrow stromal cells from Osteogenesis Imperfecta patients. BMC Med. Genet., 2014, 15, 45 .

[71] Zhang, J.; Tu, Q.; Bonewald, L.F.; He, X.; Stein, G.; Lian, J.; Chen, $\mathrm{J}$. Effects of miR-335-5p in modulating osteogenic differentiation by specifically downregulating Wnt antagonist DKK1. J. Bone Miner. Res., 2011, 26(8), 1953-1963.

[72] Qiu, W.; Kassem, M. miR-141-3p inhibits human stromal (mesenchymal) stem cell proliferation and differentiation. Biochim. Biophys. Acta, 2014, 1843(9), 2114-2121.

[73] Wang, Q.; Cai, J.; Cai, X.H.; Chen, L. miR-346 regulates osteogenic differentiation of human bone marrow-derived mesenchymal stem cells by targeting the Wnt/beta-catenin pathway. PLoS One, 2013, 8(9), e 72266.

[74] Grünhagen, J.; Bhushan, R.; Degenkolbe, E.; Jäger, M.; Knaus, P.; Mundlos, S.; Robinson, P.N.; Ott, CE. MiR-497 195 cluster microRNAs regulate osteoblast differentiation by targeting BMP signaling. J. Bone Miner. Res., 2015, 30(5), 796-808.

[75] Kureel, J.; Dixit, M.; Tyagi, A.M.; Mansoori, M.N.; Srivastava, K.; Raghuvanshi, A.; Maurya, R.; Trivedi, R.; Goel, A.; Singh, D. miR-542-3p suppresses osteoblast cell proliferation and differentiation, targets BMP-7 signaling and inhibits bone formation. Cell. Death Dis., 2014, 5, e1050.

[76] Hwang, S.; Park, S.K.; Lee, H.Y.; Kim, S.W.; Lee, J.S.; Choi, E. K.; You, D.; Kim, C.S.; Suh, N. miR-140-5p suppresses BMP2mediated osteogenesis in undifferentiated human mesenchymal stem cells. FEBS Lett., 2014, 588(17), 2957-2963.

[77] Li, Z.; Rana, T.M. Therapeutic targeting of microRNAs: current status and future challenges. Nat. Rev. Drug Discov., 2014, 13(8), 622-638. 